\title{
Pricing 50ETF in the Way of American Options Based on Least Squares Monte Carlo Simulation
}

\author{
Shuai Gao ${ }^{1}$, Jun Zhao ${ }^{1}$ \\ ${ }^{1}$ School of Science, Nanjing University of Science and Technology, Nanjing, People's Republic of China \\ Correspondence: Shuai Gao, School of Science, Nanjing University of Science and Technology, Nanjing, 210094, \\ People's Republic of China.
}

Received: May 4, 2016

doi:10.11114/afa.v2i2.1657

\author{
Accepted: May 31, 2016 \\ Available online: June 3, 2016
}

URL: http://dx.doi.org/10.11114/afa.v2i2.1657

\begin{abstract}
50ETF appears on the Chinese stock market on 9th February,2015, the contracts are European Options and the options are priced by B-S model.50ETF is the only one option that can be traded, there are no American Options in Chinese stock market. This paper studies 50ETF pricing analysis in accordance with the way of American Option. We use Least Squares Monte Carlo Simulation to price 50ETF and analyze them, give the numerical results by matlab program. This issue is worth studying, because the paper studies 50ETF, and price it in the way of American Options, we try to employ Monte Carlo Simulation to solve this problem in china and the results of the paper can enrich the option products in the stock market of China.
\end{abstract}

Keywords: 50etf, American option, least squares monte carlo simulation

\section{Introduction}

The negotiable securities of SSE 50 exchange-traded Index securities investment funds are called '50ETF', Stock code:510050,Huaxia Fund Management company is the fund manager, it is a financial product that based on a certain type of underlying assets.50ETF Option, its underlying assets are Huaxia 50ETF(510050).50ETF appears on the market on 9th February,2015 and it is the only one option that can be traded in Chinese stock market.50ETF is the only one option that can be traded, there are no American Options in Chinese stock market, in this paper, we study 50ETF, we use Least Squares Monte Carlo Simulation to price 50ETF, the price is valuable and the results can give some reference to the Chinese stock market, so the researchers can design more financial derivatives and it is also meaningful for the development of options in China.

The earliest option pricing theory was proposed by Bachelier (1990),then 65 years later, Samuelson (1965) reconsidered the problem of option pricing, he proposed the underlying assets follow Gometric Browian Motion.Then Black-Scholes (1973) and Merton (1973) studied risk neutral pricing, the famous model B-S model was created by them. Then Sharp (1978) studied perivative securities pricing, according to the self-financing strategy, Binomial tree method can price options very well. Wiggins (1983) generalized the Black-Scholes-Merton model. The models were proved price European Options very well, American options pricing was under consideration all the time. Until 1990s,Boyle, Broadie, and Glasserman (1997) given the Monte Carlo method. Longstaff and Schwartz (2001) proposed the Least Squares Monte Carlo simulation, so the option pricing theory has developed very well.

In practice, Monte Carlo simulation method is very suitable for pricing the options that are path-dependent or multivariate related, because the traditional Monte Carlo simulation uses forward solution method, at this point, we can not calculate the expected return of the options at every moment, the options are continued to hold. So the proceeds of the options that exercise immediately and the expected return of the options that continue to hold can not be compared, and we cannot decide when to exercise the options. Therefore, at the beginning, Monte Carlo simulation method is considered that can not be used for pricing American options, with the development of mathematical finance, Monte Carlo simulation has developed, there appears some Monte Carlo simulation algorithm simulation of American Option pricing. Since the 1990s, dynamic programming principle and digitmap analysis are leaded into Monte Carlo simulations, then American options are successfully priced.

The rest of the paper is structured as follows. We introduce some steps of Least Squares Monte Carlo simulate option 
pricing in Section 2.In Section 3, we price 50ETF in the way of American Options and give analysis of the results simulated by matlab program.

\section{Least Squares Monte Carlo Simulation}

As a result of the introduction of new securities and new sources of financing, various innovative techniques have been used by researchers and traders in order to determine the price of the securities (Ansari \& Riasi, 2016; Riasi, 2015). However, one of the most effective methods for pricing the securities is least squares Monte Carlo simulation.

When pricing American Options, it is necessary that make a choice whether to continue to hold options or to exercise the options on the lead time. The resulting value of exercising option is usually easy to get, some researchers, including Longstaff and Schwartz, in 2001, they proposed to choose the best match between the value of option that continue to hold and certain related variables by the Least Squares method. The Monte Carlo method has become the standard method for pricing American options, its basic principle: In a limited discrete time point, simulate the data on sample path at each time according to the underlying asset price. Obtain the expected return of the options that continue to hold by Least Squares method, comparing it and the proceeds of the options that exercise immediately, if the former is less than the latter, exercise the options immediately, else if the former is bigger than the latter, continue to hold the options.

Some steps of Least Squares Monte Carlo simulate option pricing

Firstly, generate the sample price path of the underlying asset

$$
S(t+T)=S(t) e^{\left(\mu-\frac{\sigma^{2}}{2}\right) T+\sigma t_{i} \sqrt{T}}
$$

Secondly, solve the option inversely from the expiration date,obtain the optimal execution time and option earnings on each path, at time $i$,calculate the optimal execution time and option earnings on each path.For example,put option,its intrinsic value on sample path is

$$
I_{i}^{j}\left(S_{i}^{j}\right)=\max \left(X-S_{i}^{j}, 0\right)
$$

among them, $S$ is the asset prices of sample path $j, i$ is the executive time, $X$ is executive price. Due to American option can be exercised in advance, when choose the optimal execution time, it is necessary to compare the expected return of the options that continue to hold and the instant earnings of the options that exercise in advance, choose the bigger one

$$
f_{i}^{j}\left(S_{i}^{j}\right)=\max \left(I_{i}^{j}\left(S_{i}^{j}\right), E\left(e^{-r \Delta t} f_{i+1}^{j}\left(S_{i+1}^{j}\right) \mid S_{i}^{j}\right)\right)
$$

Here, the expected return is the earnings of the options that continue to hold on the contidion $S$, regression method can give a sample polynomial about the current price of the underlying asset $S$,normally, quadratic polynomial, and use it to calculate the expected return approximately, that is

$$
E\left(e^{-r \Delta t} f_{i+1}^{j}\left(S_{i+1}^{j}\right) \mid S_{i}^{j}\right)=a\left(S_{i}^{j}\right)^{2}+b S_{i}^{j}+c
$$

$S$ is regarded as the value of the horizontal axis, let the future earnings on the path be the value of the vertical axis, then can get the coefficient analogously about the above equation by Least Squares.

Starting from the due date, obtain the optimal execution time and option earnings on each path.

On the due date, for the American put options, the options are exercised when the options become a premium, the earnings are $\max (X-S, 0)$, time $N$ is the due data, and continue to analyze the time $N-1$, if the option becomes a premium on the sample path, compare the expected return of the options that continue to hold up to the due date and the proceeds of the options that exercise immediately, if the former is less than the latter, exercise the options immediately, else if the former is bigger than the latter, continue to hold the options. The option can be exercised when it becomes a premium, it is a prerequisite, at time $N-1$, just analyze the data on the sample path, the option is a premium, the expected return of the options that continue to hold can be got by Least Squares analogously

$$
E\left[y_{N-1}^{j}\right]=e^{-r \Delta t} \max \left(X-S_{N}^{j}, 0\right)=a\left(S_{N-1}^{j}\right)^{2}+b S_{N-1}^{j}+c
$$


Therefore, when deciding whether to exercise the option in advance, only compare the expected return of the options that continue to hold and its intrinsic value at that time. Similarly, the expected return of the options that continue to hold can also be obtained at the other time. The options on the sample path can be exercised at the only time or not be exercised.

From the initial state, consider the time $\mathrm{N}$, if the optimal execution time is time $N$, at time $N-1$, if exercise the option, so the optimal execution time is time $N-1$, else the optimal execution time will not be changed, repeat the steps above. Due to every path has only one optimal execution time, finally, the expected return of the options will be got.

Thirdly, the expected return of the options on every sample path can be discounted by the risk-free rate, then take the mean, so the expected return of the options will be got, after simulating the option price many times, the optimal execution time and option earnings on each path can be obtained, finally, take the mean, so the American option price can be simulatly by Least Squares Monte Carlo Simulation.

\section{The Study of 50ETF by American Options Pricing}

\subsection{Background}

The negotiable securities of SSE 50 exchange-traded Index securities investment funds are called '50ETF', Stock code: 510050, Huaxia Fund Management company is the fund manager, the option just like the common option we know, it is a financial product based on a certain type of underlying assets.50ETF Option, its underlying assets are Huaxia $50 \mathrm{ETF}(510050)$,one contract of 50ETF option represents

10000 Huaxia 50ETF.50ETF appears on the market on 9th February, 2015, call option; put option, four expiry times, five prices that the option be exercise, 40 contracts in total. The expiry time is March, April, June and September. The closing price of 50ETF is 2.291 yuan on 6th February, 2015. According to the spacing of exercising the options, five strike price of the options are 2.20 yuan, 2.25 yuan, 2.30 yuan, 2.35 yuan and 2.40 yuan.

\subsection{Data preparation (Employ Monte Carlo Simulation to price 50ETF)}

S: 2.291 yuan;

$\mathrm{K}: 2.20$ yuan, 2.25 yuan, 2.30 yuan, 2.35 yuan and 2.40 yuan;

$\mathrm{T}$ : if June or September is chosen as the expiry time, then the time will be four months or seven months, so $\mathrm{T}$ will be 0.3333 years or 0.5833 years;

r: risk-free rate, here choose the three-year treasury bonds interest rates that issues in 2015 in China, the value is $4.92 \%$;

$\sigma$ : the volatility of the underlying asset,use historical data to estimate volatility, the observation data of the stock price is usually within a fixed time, definition

$$
n+1: \text { the times of the observation data; }
$$

$S_{i}$ : the stock prices that ended at the $\mathrm{i}$-th time zone, $i=0,1, \ldots, n$;

$\tau$ :the length of time and its unit: year

$$
\text { let } u_{i}=\ln \left(\frac{S_{i}}{S_{i-1}}\right), i=1, \ldots n
$$

$$
u_{i} \text { is the estimated value of standard deviation, } s \text { is } s=\sqrt{\frac{1}{n-1} \sum_{i=1}^{n}\left(u_{i}-\bar{u}\right)^{2}} \text { or }
$$

$s=\sqrt{\frac{1}{n-1} \sum_{i=1}^{n} u_{i}{ }^{2}-\frac{1}{n(n-1)}\left(\sum_{i=1}^{n} u_{i}\right)^{2}}, \bar{u}$ is the mean of $u_{i} . \sigma \sqrt{\tau}$ is the standard deviation of $u_{i}$, so the variable $s$ is the estimated value of $\sigma \sqrt{\tau}$, and $\sigma$ itself can be estimated as $\bar{\sigma}$, here $\bar{\sigma}=\frac{s}{\sqrt{\tau}} \cdot \tau$ is the number of stock trading 
days within a year, generally, set the number of days of trading days per year 252 . The article chooses the data whose underlying asset is Huaxia 50ETF (the historical data is from 5th January,2015 to 15th Demcember,2015),use the above model, the estimated value of the volatility is 0.4097 .

\subsection{Pricing American-style Options by Monte Carlo Simulation}

3.3.1 Pricing with the expiry time is April and the strike price is 2.30 yuan

For example, the American Put Option are priced by Least Squares Monte Carlo model,April is chosen as the expiry time, the strike price is 2.30 yuan. Using the Least Squares Monte Carlo model, the accuracy of the results depend on the times that simulated,the more times, the results will be more precise, simulate the option 10 times and 1500 paths, the results are as follows

Table 1.The value of American put option and call option

\begin{tabular}{ccc}
\hline Frequency & Put option & Call option \\
\hline 1 & 0.1548 & 0.1608 \\
2 & 0.1526 & 0.1623 \\
3 & 0.1515 & 0.1586 \\
4 & 0.1551 & 0.1598 \\
5 & 0.1522 & 0.1532 \\
6 & 0.1479 & 0.1687 \\
7 & 0.1516 & 0.1441 \\
8 & 0.1509 & 0.1672 \\
9 & 0.1585 & 0.1768 \\
10 & 0.1483 & 0.1588 \\
Average & 0.1523 & 0.1610 \\
\hline
\end{tabular}

The results are simulated by the Least Squares Monte Carlo,they are random,simulate the option 10 times and take the average of the results,the value of American put option is 0.1523 yuan and the value of American call option is 0.1610 yuan.

3.3.2 Pricing American-style Options with all expiry time and strike prices

50ETF Option,four expiry time,five strike prices,study the American Put and Call Options,forty prices of the option will be obtained by matlab program.Just like 2.3.1,each price is simulated many times.

The forty results are simulated as follows:

Table 2 . The forty prices of the put and call options obtained by matlab program

\begin{tabular}{lccccccccc}
\hline $\begin{array}{l}\text { Time } \\
\begin{array}{c}\text { Strike } \\
\text { Price } \\
\text { Yuan })\end{array}\end{array}$ & Put & Call & Put & Call & Put & Call & Put & Call \\
\hline 2.20 & 0.0631 & 0.1666 & 0.1022 & 0.2119 & 0.1564 & 0.2957 & 0.2133 & 0.3881 \\
& & & & & & & & \\
2.25 & 0.0839 & 0.1405 & 0.1254 & 0.1805 & 0.1776 & 0.2458 & 0.2366 & 0.3345 \\
2.30 & 0.1056 & 0.1089 & 0.1523 & 0.1610 & 0.2032 & 0.2200 & 0.2642 & 0.3157 \\
2.35 & 0.1371 & 0.0911 & 0.1771 & 0.1404 & 0.2357 & 0.2034 & 0.2901 & 0.3050 \\
2.40 & 0.1661 & 0.0712 & 0.2049 & 0.1164 & 0.2605 & 0.1790 & 0.3110 & 0.2777 \\
\hline
\end{tabular}

The forty prices of the options are obtained by matlab program. To get precise results, each price is simulated more than 
10 times by matlab program, so the price is valuable and the results can give some reference to the Chinese stock market, so the researchers can design more financial derivatives and it is also meaningful for the development of options in China.

3.3.3 Analysis and comments

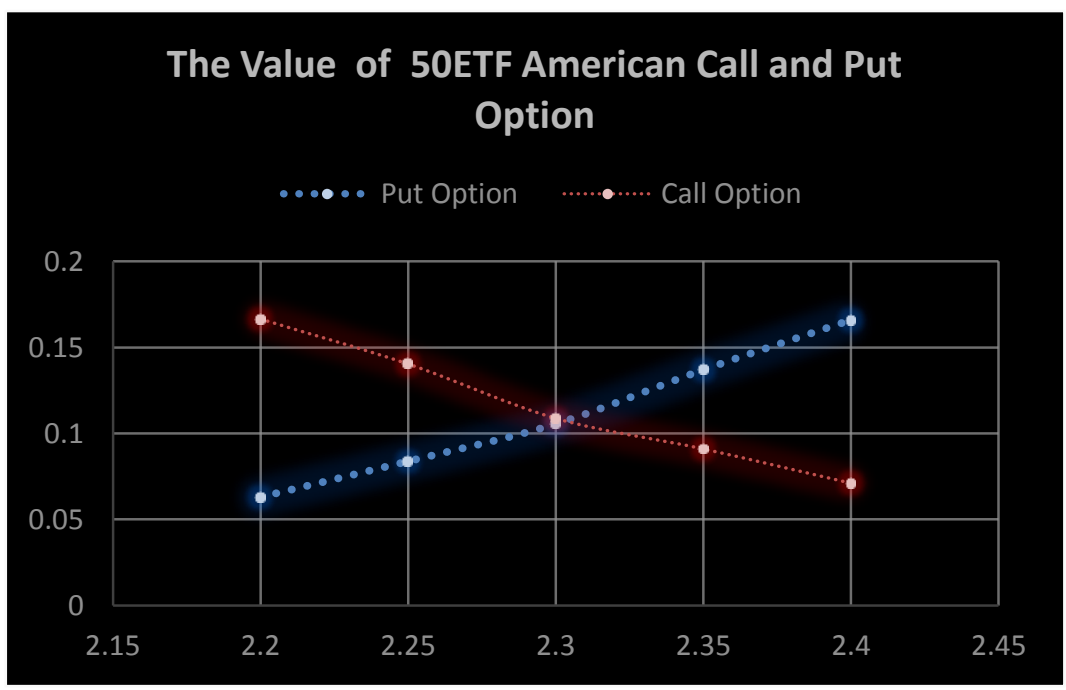

Chart 1 . The value of 50ETF options change with the stike price K,March is chosen as the expiry time

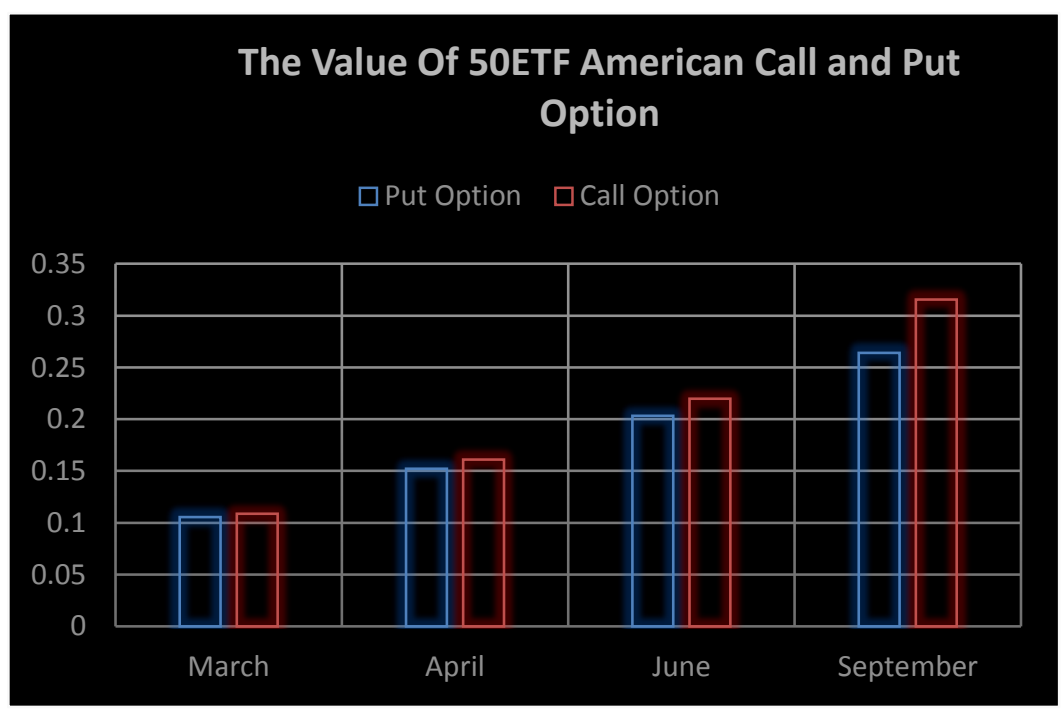

Chart 2. The value of 50ETF options change with the expiry time $\mathrm{T}$, the stike price is 2.30 yuan

For the American Option, five factors affect the value of the option: the price of the underlying asset, the strike price, the expiry time, the volatility of the underlying asset and risk-free rate. Seeing from the forty results above, under the same conditions, with the increase of strike price, the value of the American Put Option will increase while the value of the American Call Option will decrease, the Chart 1 can explain this phenomenon; similarly, under the same conditions, with the increase of time, both of the value of the American Put Option and Call Option will increase, the Chart 2 can explain this phenomenon.

In fact, many methods can price options, Monte Carlo simulation method is very suitable for pricing options that are path-dependent or multivariate related. If pricing American-style Options By Monte Carlo Simulation, the results may be random, it is necessary to simulate many times to make sure that the results precise. So the Monte Carlo Simulation can be improved to cut down randomness, it needs further study. 


\section{Conclusion}

This paper studies 50ETF pricing analysis in accordance with the way of American Option. We use Least Squares Monte Carlo Simulation to price 50ETF and analyze them, give the numerical results by matlab program. Alouthgh many methods can price options, generally, Monte Carlo simulation method is widely used and is very suitable for pricing American options. In Chinese stock market, 50ETF is the only one option that can be traded, there are no other options, the option is European Option. Except China, it is also a problem in some other stock markets, the paper studies 50ETF, and price it in the way of American Options, the results of the paper can give some guidance and help to researchers, it can guide them to design new financial derivatives and it is also beneficial for development of options in China.

\section{References}

Ansari, A., \& Riasi, A. (2016). An Investigation of Factors Affecting Brand Advertising Success and Effectiveness, International Business Research, 9(4), 20-30. http://dx.doi.org/10.5539/ibr.v9n4p20

Bernard, C., \& BoyleMonte, P. P. (2011). Carlo Methods for Pricing Discrete Parisian Options, 17(3), 169-196.

Boyle, P. P. (1977). Options: A Monte Carlo approach, Journal of Financial Economics, 4, 323-338.

Boyle, P., Broadie, M., \& Glasserman, P. (1997). Monte Carlo methods for security pricing, Journal of Economic Dynamics \& Control, 21(8-9), 1267-1321.

Cescato, C. D., \& Lemgruber, E. F. (2011). Valuation of American interest rate options by the Least-Squares Monte Carlo method, Pesquisa Operacional, 31(3), 521-541.

Clément, E., Lamberton, D., \& Protter, P. (2002). An Analysis of a Least Squares Regression Method for American Option Pricing, Finance \& Stochastics, 6, 449-471.

Corporation, H. P. (2014). Randomized Binomial Tree and Pricing of American-Style Options, 1, 1-6.

Jia, Q. (2010). Pricing American Options using Monte Carlo Methods, Department of Mathematics.

Liu, Q. (2008). Pricing American Options by Canonical Least-Squares Monte Carlo, Journal of Futures Markets, 30(2), $175-187$.

Longstaff, F. A. (2001). Valuing American Options by Simulation: A Simple Least-Squares Approach, Review of Financial Studies, 14, 113-147.

Riasi, A. (2015). Competitive Advantages of Shadow Banking Industry: An Analysis Using Porter Diamond Model, Business Management and Strategy, 6(2), 15-27. http://dx.doi.org/10.5296/bms.v6i2.8334 .

Wu, Z. (2012). Pricing American Options using Monte Carlo Method, Numerical Analysis.

Yeung, J. A. (2010). Path-Dependent Options Pricing: A Quasi Monte Carlo Simulation Approach with MATLAB, Ssrn Electronic Journal.

Zhao, Q., Liu, G., \& Gu, G. (2013). Variance Reduction Techniques of Importance Sampling Monte Carlo Methods for Pricing Options, Journal of Mathematical Finance, 4, 431-436

\section{$(\mathrm{cc}) \mathrm{BY}$}

This work is licensed under a Creative Commons Attribution 3.0 License. 